\title{
Entre diabolisation, séduction et légitimation. Le kitsch ou l'imitation comme « mal esthétique »?
}

Zwischen Verteufelung, Verführung und Legitimierung: Der Kitsch oder die Nachahmung als „ästhetisches Übel"?

Between demonisation, seduction and legitimation: kitsch or imitation as "aesthetic evil"?

Florence Bancaud

\section{(2) OpenEdition}

Journals

Édition électronique

URL : http://journals.openedition.org/ceg/336

DOI : $10.4000 /$ ceg.336

ISSN : 2605-8359

\section{Éditeur}

Presses Universitaires de Provence

Édition imprimée

Date de publication : 5 avril 2017

Pagination : 73-88

ISBN : 979-10-320-0103-5

ISSN : 0751-4239

\section{Référence électronique}

Florence Bancaud, «Entre diabolisation, séduction et légitimation. Le kitsch ou l'imitation comme « mal esthétique »? », Cahiers d'Études Germaniques [En ligne], 72 | 2017, mis en ligne le 05 octobre 2018, consulté le 25 novembre 2020. URL : http://journals.openedition.org/ceg/336 ; DOI : https:// doi.org/10.4000/ceg.336 


\title{
Entre diabolisation, séduction et légitimation Le kitsch ou l'imitation comme " mal esthétique "?
}

\author{
Florence BANCAUD \\ Aix-Marseille Université
}

\begin{abstract}
Splendide Tour Eiffel à paillettes bleues [...], belle Vierge Marie pleine d'eau bénite (...), élégante Vénus de Botticelli en résine et en promotion à saisir (...), éclatante robe de bal en rayonne verte agrémentée de nouds et de rubans rose fuchsia (....). Le kitsch est partout, en tout. Il décore, il orne, il enrubanne, le tout dans une bonne conscience de soi. Il ajoute du mignon, du mignard, de la fanfreluche, comme si la vie était plus légère à porter avec cette parure insistante [...]. Il diffuse son mauvais goût apparent dans tous les aspects de notre existence par son sentimentalisme facile et son imagerie stéréotypée. De la pacotille comme art de vivre. Il ravale tout effort de sincérité au rang de camelote vite échangée. Le kitsch commence par un coup d'éclat mais finit comme un dégonflement ${ }^{1}$.
\end{abstract}

Si le kitsch nous intéresse ici, c'est que, partant d'une " imitation, d'une pièce inauthentique pour en faire un objet de parade ${ }^{2}$ " et fondé sur la reproduction et la réadaptation de modèles ou de stéréotypes pour un public de masse, il constitue une donnée fondamentale du changement qui s'opère dans l'inconscient visuel au XIX ${ }^{\mathrm{e}}$ siècle. De fait, la reproduction sérielle des produits manufacturés crée une nouvelle forme de sensibilité moderne fondée sur la répétition en série et sur la collection. En témoignent la nouvelle popularité des daguerréotypes dans les années 1830, puis l'essor des albums photos à partir des années $1850^{3}$ : la nouveauté et la multiplicité des copies se substituent ainsi aux valeurs d'authenticité et d'unicité. Or, le principe qui sous-tend l'authenticité est que " seul l'objet original puisse transmettre ses significations purement et irrévocablement. Lorsque l'objet est reproduit, sa signification est déformée, voire irrémédiablement perdue. Vidées de l'essence originelle, les copies sont ravalées

1. Christophe Genin, Kitsch dans l'âme, Paris, Vrin, 2010, p. 9.

2. Ibid., p. 81.

3. Voir Céleste Olalquiaga, Royaume de l'artifice. L'émergence du kitsch au XIXe siècle, New York, Pantheon Books, 1998, p. 14-17. 
au rang de pâles imitations et opposées à l'objet authentique ${ }^{4}$ ". Baudrillard définit ainsi le kitsch comme " pseudo-objet " ou équivalent du " cliché ${ }^{5}$ " dans le discours, parlant d'une esthétique de la simulation propre au kitsch, qui, sans jamais innover, "se défınit par sa valeur dérivée et pauvre ", " reproduit les objets plus petits ou plus grands que nature ", "imite les matériaux ", " singe les formes " et "répète la mode sans l'avoir vécue ${ }^{6}$ ".

Quel est donc le statut de l'imitation dans le kitsch? L'imitation dévaluet-elle son objet en faisant de l'art un simple vecteur de plaisir et un objet de consommation? En se vouant au culte du bel effet, en quoi le kitsch peut-il être considéré comme un contre-modèle de l'art véritable? Mais ne peut-on pas aussi y voir un jeu postmoderne avec la tradition, où le principe d'ironie et de décalage peut contribuer à en faire une nouvelle catégorie esthétique à part entière?

\section{Le kitsch comme principe d'imitation et de dévalorisation}

Le principe de reprise et d'imitation est inscrit au cœur du kitsch. Rappelons qu'il s'agit d'un terme d'origine bavaroise né en Allemagne vers 1860 du verbe verkitschen qui dérive de "kitschen " et signifie ramasser des déchets dans la rue, bâcler, faire de nouveaux meubles avec du vieux, voire vendre de la camelote à la place de ce qui a été effectivement demandé. Le kitsch désigne des objets et du mobilier peu coûteux de style Biedermeier prisé par les petits employés et les ouvriers ${ }^{7}$ car ressemblant au mobilier de grand style tout en restant abordables: dans les cercles artistiques munichois, note aussi Christophe Genin, le terme aurait aussi désigné vers 1875 une image de piètre qualité, bon marché, un cliché surchargé ou un objet culturel trivial ${ }^{8}$.

L'apparition du mot est donc liée à un tournant sociologique; produit de la civilisation bourgeoise et de l'industrialisation à outrance du XIX ${ }^{\mathrm{e}}$ siècle, le kitsch répond aux nouveaux besoins de la culture de masse qui se développe alors; accédant au marché des objets de décoration, les classes moyennes " prises entre le besoin de s'identifier aux classes supérieures par des signes extérieurs de réussite sociale et des revenus insuffisants pour se constituer un patrimoine d'adoption, s'orientent vers la copie, l'imitation" ", ce qui provoque une crise de la culture aristocratique. Instrument de promotion de la classe bourgeoise, au moment où elle adopte le caractère d'" affluence ", c'est-à-dire l'excès des moyens sur les besoins, et offrant une " ostentation à bas prix ${ }^{10}$ ", le kitsch

\footnotetext{
4. Ibid., p. 18.

5. Jean Baudrillard, La société de consommation, Paris, Denoël, 1970, p. 165.

6. Ibid., p. 168.

7. Catherine Coquio, "Kitsch et critiques du kitsch: spleen du Beau et mal de l'art ", in L'art contre l'art. Baudelaire, le " joujou " moderne et la "décadence ", Bandol, Vallongues, 2005, p. 311.

8. Genin, Kitsch dans l'âme, p. 10.

9. Ibid., p. 33.

10. Ibid., p. 12-13.
} 
devient alors un art du faux, dès lors que l'objet d'art, voué à la consommation, devient une marchandise destinée à la masse. C'est un art du bel effet immédiat où l'objet opère comme un fétiche voué à manifester une puissance, à satisfaire un désir d'appropriation et de valorisation sociale plus qu'à satisfaire le sens esthétique.

Le paradis du kitsch est le grand magasin comme le Bon Marché d'Aristide Boucicaut décrit en 1852 par Zola comme un véritable temple de commerce dans le Bonheur des Dames: espace ouvert et séduisant, aux vitrines immenses et illuminées, aux prix affichés en toute transparence, le grand magasin marque le triomphe de la démocratie bourgeoise du commerce où tous sont égaux devant l'achat; pris du vertige de la consommation, le client se croit dominant et non plus dominé par le commerçant méfiant d'autrefois : dans les temples du commerce moderne, "l'esprit est remplacé par l'impulsion, le besoin par le désir, le calcul par la munificence, la sémantique par l'esthétique ${ }^{11}$ ".

Ainsi la Nana de Zola aime-t-elle à flâner dans le passage des panoramas où elle se remémore les plaisirs passés :

Elle adorait le passage des Panoramas. C'était une passion qui lui restait de sa jeunesse pour le clinquant de l'article de Paris, les bijoux faux, le zinc doré, le carton jouant le cuir. Quand elle passait, elle ne pouvait s'arracher des étalages [...], prise surtout par le goût criard des bibelots à bon marché, [...] des coquilles de noix, des hottes de chiffonnier pour les cure-dents, des colonnes Vendôme et des obélisques portant des thermomètres ${ }^{12}$.

L’imitation aboutit ici à un processus de dévaluation de l'aristocratisme: " 'on' en emprunte les valeurs, non pour se les approprier comme signe d'appartenance, mais pour les copier comme signe extérieur de promotion ou de réussite ${ }^{13}$ ". Mais cette imitation, loin d'être fidèle à l'original, singe à contretemps l'objet d'art ou le style qu'elle déforme, exacerbe ou simplifie pour plaire à la masse, offrant ainsi une " ostentation à bas prix ${ }^{14}$ " et résultant donc d'une vulgarisation commerciale et esthétique de l'œuvre ou de l'objet d'art ${ }^{15}$.

Objet produit en série ${ }^{16}$, périssable, produit et vécu dans l'insouciance, l'objet kitsch n'aspire pas à la pérenne solennité de l'œuvre, mais se nourrit de la copie, de la production et de la reproduction en masse d'objets industriels ${ }^{17}$. Thorstein Veblen, dans sa Théorie de la classe de loisir ${ }^{18}$, dénonçait déjà en 1899 cette consommation ostentatoire comme gaspillage de temps et de biens qui,

11. Voir Abraham Moles, Psychologie des Kitsches, München, Hanser, 1971, p. 91.

12. Émile Zola, Nana [1880], Paris, Le Livre de Poche, 1956, p. 221.

13. Ibid., p. 40-41.

14. Genin, Kitsch dans l'âme, p. 13.

15. Coquio, "Kitsch et critiques du kitsch ", p. 312.

16. Dans une lettre à Victoria Ocampo datant de 1934, Drieu la Rochelle critiquait lui-même la fabrication sérielle, affirmant: "Nous sommes malades comme nos objets fabriqués en série. Et nous fabriquons en série - puisque nous fabriquons pour fabriquer. " Cf. Pierre Drieu La Rochelle, Victoria Ocampo, Lettres d'un amour défunt. Correspondance 1929-1944, édition établie par Julien Hervier, Paris, Bartillat, 2009.

17. Genin, Kitsch dans l'âme, p. 12.

18. Thorstein Veblen, Théorie de la classe de loisir, Paris, Gallimard (coll. " Tel »), 1978. 
substituant à l'original une copie qui " fait le même effet, à moindre coût ", réduit celle-ci au rang d'objet réifié, de relique figée dans une éternité artificielle.

Le kitsch désigne donc moins un objet qu'un mode de réception des objets et de l'art qui engendre une perversion du goût et de l'art lui-même. C'est pourquoi il fait l'objet d'un jugement de valeur souvent négatif opposant à ce qui est beau, distingué, noble, sérieux, et original la légèreté, la vulgarité, la trivialité, la banalité, la charge et la lourdeur parfois ridicule et chargée de clichés ainsi que l'absence d'âme.

\section{Le kitsch comme contre-modèle de l'art}

Dès 1939, dans son célèbre essai Avantgarde and Kitsch, Clement Greenberg défınissait le kitsch comme culture de l'ersatz et du simulacre, par opposition à l'art d'avant-garde, affirmant que si l'art d'avant-garde imite le geste créateur divin, le kitsch est " imitation de l'art d'imiter ", ou " imitation des effets de l'imitation " ${ }^{19}$. Dans les années 1970, les essais de Dorfles (1968), Giez (1971) ou Moles (1971) sur le kitsch l'analysent dans une perspective psychosociologique comme anti-art fondé sur les principes d'accumulation et d'inadéquation.

Abraham Moles, dans sa célèbre Psychologie du kitsch ${ }^{20}$, voit avant tout dans le kitsch un style d'absence de style désignant non seulement un processus de production, mais aussi une attitude esthétique et artistique, une manière d'être liée à l'insertion dans la vie de valeurs bourgeoises. L'attitude kitsch, décrite comme un " phénomène social universel, permanent et de grande envergure ", désigne un type de rapport que l'être entretient avec les choses, un état d'esprit qui se cristallise dans les objets: les rapports de l'individu bourgeois avec le milieu social passant désormais fondamentalement par les objets, le phénomène kitsch est fondé sur une civilisation consommatrice qui produit pour consommer et crée pour produire. Le mode de relation de l'homme kitsch avec son entourage matériel est désormais fondé sur l'appropriation de l'objet, que celle-ci se décline sur le mode du fétichisme pour le collectionneur, de l'insertion de l'objet dans un ensemble chez le décorateur ou de la collection chez l'amateur d'art. Mais dans tous les cas, la singularité de l'objet se voit gommée par la copie en masse de produits et par l'accélération consommatrice qui fait de l'objet un moment transitoire de l'existence d'un multiple pris entre la fabrique et la poubelle.

19. Clement Greenberg, "Avantgarde and kitsch ", en ligne: [http://www.sharecom.ca/greenberg/ kitsch.html]: "The avant-garde poet or artist tries in effect to imitate God by creating something valid solely on its own terms [...], something given increate, independant of meanings, similars or originals (...). What we have here is the imitation of imitating [...] (...). Kitsch is mechanical and operates by formulas. Kitsch is vicarious experience and faked sensations. Kitsch changes according to style, but remains always the same [...]. If the avant-garde imitates the processes of art, kitsch, we now see, imitates its effects. "

20. Abraham Moles, Psychologie du kitsch, Paris, Denoël / Gonthier, 1971 / Psychologie des Kitsches, München, Hanser, 1971. 
La société de masse fait ainsi du milieu quotidien un flux permanent plutôt qu'un sédiment durable.

L'objet kitsch, banal, démocratisé, incarne alors une forme d'art agréable, non subversif, à la mesure de l'homme; il génère le plaisir par la communion secrète dans un " mauvais goût " reposant et modéré, vertu du milieu médiocre. L'idée de la beauté est remplacée par celle du plaisir lié au sensuel, et surtout au consensus d'une majorité sociale (la convenance). Mais de par son ancrage dans le quotidien et dans le banal, l'aliénation en est un des facteurs essentiels. Les choses de Perec montrent très bien comment cette aliénation s'installe à travers les objets, dans une possession acquisitive, mais transitoire, chez un couple de Parisiens préparés par leur métier dans le marketing à la perception de cette aliénation, mais en même temps victimes impuissantes de celle-ci :

Ils rêvaient de porcelaines précieuses, à décors d'oiseaux exotiques, de livres reliés de cuir [...] de tables d'acajou, de vêtements de soie et de lin, souples et confortables, pleins de couleurs, de chambres spacieuses et claires, de brassées de fleurs, de tapis de Boukhara, de dobermans bondissants [...].

Mais ils étouffaient sous l'amoncellement de détails. Les images s'estompaient, se brouillaient [...], comme si ces images n'avaient jamais été que des reflets très lointains, démesurément obscurcis, des scintillations allusives, illusoires, qui s'évanouissaient à peine nées, des poussières $^{21}$.

Fondé sur l'imitation et la dégradation de l'art noble, le kitsch se nourrit par défınition de la surcharge et de l'excès d'ornement: si les courbes en régissent les contours et les éléments, les objets kitsch comportent des surfaces généralement pleines ou enrichies de symboles ou d'ornements souvent outranciers; les couleurs sont contrastées, allant du rose bonbon fondant au violet et au lilas laiteux et jouant sur les couleurs non franches. Les matériaux sont rarement utilisés pour ce qu'ils sont: ainsi le bois peint en imitation marbre, le plastique avec fibres incorporées, les zincs bronzés, (les) bronzes dorés qui, tous, visent à simuler l'authentique.

Les principes de l'assemblage d'objets kitsch sont l'entassement d'objets divers dans un volume à la superficie restreinte au risque de resserrer l'espace vital et de comprimer celui-ci - c'est la " pression kitsch " dont parle Moles; l'hétérogénéité ensuite; les objets assemblés en vertu d'un " surréalisme combinatoire inconscient ${ }^{22}$ " n'ont pas de rapport direct les uns aux autres; l'anti-fonctionnalité enfin, les objets ne répondant pas à un critère utilitaire.

Les caractéristiques du kitsch relevées par Moles sont donc: l'inadéquation de l'objet par rapport à la fonction qu'il est censé remplir; le kitsch " vise toujours un peu à côté, il remplace le pur par l'impur ${ }^{23}$ ", aboutissant ainsi à une sur- ou à une sous-dimensionnalisation de l'objet. L'accumulation dans l'idée d'encombrement ou de frénésie du "toujours davantage " qui émerge

21. Georges Perec, Les Choses, Paris, Gallimard, 1965.

22. Moles, Psychologie des Kitsches, p. 62.

23. Ibid., p. 61 
dans la civilisation bourgeoise ${ }^{24}$; la perception synesthésique visant à assaillir le plus possible de canaux sensoriels simultanément dans une volonté d'art total $^{25}$; la médiocrité : à travers cette accumulation de moyens, le kitsch reste essentiellement un art de masse, un art du plaisir facile, de la sécurité fondée sur l'accumulation et la conservation de capital, un art de la Gemütlichkeit liée au " confort de l'âme et du cœur ${ }^{26}$ ".

Le kitsch, opposé à la modernité des avant-gardes et au fonctionnalisme ${ }^{27}$, naît donc de l'art du " néo ", fondé sur l'imitation comme valeur fondamentale: un art qui « se cherche sur l'héritage des valeurs du passé, acceptées sans examen comme belles, par une culture bourgeoise récemment promue " et qui se satisfait de la " copie avec variations ${ }^{28}$ ", le néo se distinguant seulement de l'ancien par un matériau et un style nouveaux, mais s'opposant à toute idée de nouveauté ou d'avant-garde.

Le roi du kitsch est évidemment Louis II de Bavière, roi de l'ersatz néo-authentique. Adulant le Roi-Soleil, il fait construire de 1868 à 1878 son palais de Herrenchiemsee comme une réplique au château de Versailles; mais si ce dernier est " l'accomplissement de la raison, de la maîtrise de la nature ", Herrenschiemsee marque le triomphe de la surcharge: sa galerie des Glaces mesure $98 \mathrm{~m}$ de long contre les $73 \mathrm{~m}$ de Versailles, brisant l'harmonie classique conçue par Mansart, et créant un effet de surcharge avec les stucs-marbres des murs, les tapisseries peintes en trompe-l'œil qui réduisent le palais à un décor de théâtre clinquant qui confıne au mauvais goût. Le kitsch tend ici au ridicule conçu comme déchéance du sublime dont il « perd l'élévation pour ne garder que la surcharge ${ }^{29} "$; de même, le style Biedermeier créé entre 1820 et 1850, style anti-aristocratique et antipopulaire qui se nourrit de la copie permanente de lui-même et qui, à partir de l'objet-meuble, constitue " cet intérieur kitsch tout pénétré de Gemütlichkeit où les porcelaines de Meissen, Limoges, Sèvres ou de Pékin s'entassent sans usage, objets faits pour être regardés, peuplant de bibelots le salon ${ }^{30}$."

Les années 1880-1914 marquent également le triomphe du kitsch dans une littérature en quête d'absolu, mais qui oppose à l'aspiration à l'infini des romantiques le désir d’installation et de sécurité dans la " coquille bourgeoise ${ }^{31}$ ":

24. Dans son Essai sur Wagner de 1937, Adorno souligne aussi la théâtralité kitsch des figures de Lohengrin, Parsifal, Siegfried, l'emphase du geste de l'acteur figé en image expressive, et les tableaux wagnériens comme manière d'exposition des topoi romantiques.

25. Moles, Psychologie des Kitsches, p. 64.

26. Ibid., p. 65.

27. "L'avant-garde revendique l'inchoatif et le kitsch, qui réagence des modèles anciens, procède par itération. ", in: Anne Beyaert-Geslin, "Kitsch et avant-garde: de l'objet à la stratégie culturelle ", Actes sémiotiques, publié en ligne le 24 janvier 2007 : http ://epublications.unilim. $\mathrm{fr} /$ revues/as/3239

28. Eberhard Wahl, Abraham Moles, "Kitsch et objet ", Communications 13, 1969, p. 105-129 / http//perseee.fr/web/revues/home/prescript/article/comm_0588-8018_1969_num_13_1_1188

29. Cf. Genin, Kitsch dans l'âme, p. 24-25.

30. Eberhard Wahl, Abraham Moles, "Kitsch et objet ".

31. Ibid. 
le kitsch littéraire parle de nobles héros, de vierges évanescentes, de vieillards à barbe blanche; il procède par accumulation et par répétition, usant à l'envi de l'enflure et de la surcharge: sur la table, l'auteur kitsch met une nappe, sur la nappe un plateau, sur le plateau un napperon, sur le napperon des soucoupes, puis des tasses; l'héroïne n'habite pas seulement au bord de la mer, mais dans une ville blanche sous des pins parfumés, aux bords d'une mer argentée, sous la lumière de la lune. Dans son anthologie Deutscher Kitsch, Walter Killy montre ainsi comment la littérature kitsch use du stéréotype, d'associations banales (la femme belle et pauvre, le serviteur fidèle, l'enfant égaré, l'ami loyal) et recourt à des systèmes d'association automatiques : le voile est brodé d'or, le mur immense, les palmes bruissent dans le luxe; un concept normal y est toujours poussé à l'extrême, le joli devenant beau, le riche très riche, le laid horrible.

\begin{abstract}
Adolphine streckte ihre zarten Glieder auf das weiche Moos; das heilige Rauschen in den Wipfeln der uralten Bäume, das Plätschern des zum Vater Rhein hinabeilenden Baches, lullten die Schlummermüde ein. Der Champagner und die Freude hatten den Liliensammet ihrer Wangen gerötet [...]. Freundlich lächelten die Purpurlippen, als schwebe ihr der Scherz des Tages vor der freudetrunkenen Seele, der kleine Mund war halb geöffnet, wie eine eben sich entfaltende Rosenknospe. " (A. Clauren)

Helle Wiesen gehen auf, Frühlinge spielen mit leicht bewegten Gestalten, und vor dem Herbst sitzt eine alte Frau, eine böse Frau, um die herum alle Blätter fallen. Winter wird sein. Große glänzende Engel, die den Schnee nicht streifen, aber so hoch wie die Himmel sind, werden sich zu horchenden Hirten neigen und ihnen singen von dem Märchenkinde in Bethleem. " (Rilke) ${ }^{32}$
\end{abstract}

Le kitsch musical se caractérise également par une forte disproportion entre les moyens employés et le thème: un gros orchestre jouera ainsi une mélodie banale. Et on y retrouve la cumulation des effets, et l'absence de sobriété ainsi qu'un déphasage volontaire dans la transcription de la marche nuptiale de Mendelssohn à l'accordéon ou dans la Neuvième symphonie jouée par un orchestre de brasserie ; on parlera également de " kitschisation " ( Verkitschung ") d'un morceau par sa reprise en chœur ou par son accompagnement à un registre inférieur ou avec addition d'un chœur, dans le but de provoquer la sensualisation sonore et la simplification du thème: dans tous ces cas, le kitsch combine imitation et travestissement simplificateur.

Le processus de " kitschisation ${ }^{33}$ résulte donc de l'association de l'imitation au composite: " c'est parce qu'elle est composite, c'est-à-dire qu'elle mélange des styles et des matériaux relevant d'ordres divers, que l'imitation semble fausse $^{34}$ ": il n'y a plus d'hylémorphisme, d'accord entre forme et matière et, " par ce composite, l'imitation tombe donc dans la caricature, aussi bien la déformation de l'original que la dévaluation de la copie elle-même ${ }^{35}$. " Ainsi le

32. Cité in Walter Killy, Deutscher Kitsch. Ein Versuch mit Beispielen (2 éd.), Göttingen, Vandenhoeck \&t Ruprecht, 1962.

33. Genin, Kitsch dans l'âme p. 24.

34. Ibid., p. 25.

35. Ibid. 
Sacré Cœur aux fondations en béton, au style romano-byzantin et à la coupole au byzantinisme bâtard, associé à une structure à la ligne claire.

\section{Le kitsch symptôme d'une crise d'authenticité?}

Que tue donc le kitsch? L'originalité tout d'abord. Reposant en effet sur le postulat qu'il n'y a pas de substance en soi, ni d'origine ayant valeur de distinction qualitative, le kitsch fonctionne comme un " substitut qui remplit la même fonction sans avoir les mêmes propriétés que l'original devenu obsolète, périmé, superflu. (...). Lorsque l'objet est reproduit, sa signification est déformée, voire irrémédiablement perdue. Vidées de l'essence originelle, les copies sont ravalées au rang de pâles imitations ${ }^{36}$."

Le kitsch manifeste également une crise de l'authenticité qui se constitue dans le temps long de la mémoire et dans l'" inquiétude de sa dénaturation ${ }^{37}$ ". Dans son Salon de 1846, Baudelaire l'associe au chic et au poncif, marque du temps travaillant l'art lui-même, mais aussi signe de l'abus de mémoire des caractères et des formes que l'artiste conventionnel se contente de recopier lorsqu'il manque d'imagination et d'originalité, restant prisonnier de sa dévotion envers une tradition dénoncée comme sclérosante.

Enfın, le kitsch témoigne de la perte d'aura de l'œuvre d'art dont l'authenticité et l'originalité sont anéanties à l'ère de la reproduction en série ; à l'ère des masses, note Walter Benjamin, le sujet revendique l'accessibilité de l'œuvre, sa prise de possession immédiate; sortant ainsi l'objet de son halo sacré, elle en détruit l'aura et ne permet plus de s'abîmer dans la contemplation de l'œuvre. Dans Kitsch onirique, Benjamin recourt à la métaphore de la poussière pour évoquer le délabrement des rêves modernes qui, parallèlement à cette " désauratisation " de l'art, deviennent tangibles et proches, perdant leur dimension romantique d'échappées imaginaires pour entrer dans l'atmosphère polluée de la vie quotidienne $\|^{38}$. Le kitsch témoigne de cette fétichisation de l'œuvre d'art qui sombre dans le banal et le familier et dont la valeur d'exposition refoule la valeur rituelle.

Le mode de perception aussi en est transformé, passant d'une réception contemplative et individuelle à une réception collective et simultanée où la jouissance esthétique se fait sur le mode de la distraction passive; Benjamin souligne pour cette raison le danger d'esthétisation des masses et de la politique par le fascisme, concluant son essai par le célèbre adage qu'à l'esthétisation de la politique par le totalitarisme fasciste, il faut répondre par une politisation de l'art.

36. Olalquiaga, Royaume de l'artifice, p. 26.

37. Genin, Kitsch dans l'âme,, p. 14.

38. Walter Benjamin, Kitsch onirique (1927), Euvres II, trad. Pierre Rusch, Paris, Gallimard, 2000, p. 9-10. 
Le kitsch cristallise ainsi de la crise de l'art moderne soumis au processus général de "désesthétisation ${ }^{39}$ " de l'art ou de séduction narcissique conçue par Lipovetsky ${ }^{40}$ comme principe d'organisation des sociétés d'abondance qui transforme le réel en représentation fausse, désublimant les œuvres ${ }^{41}$ et légitimant tous les sujets au nom d'un hédonisme exacerbé et substituant à l'esthétique de la contemplation une esthétique de la sensation et de l'effet immédiat.

\section{Le procès du kitsch comme décadence de l'art}

Nietzsche est un des premiers à critiquer cette vulgarisation de l'esthétique dans Le Cas Wagner où il conspue l'histrionisme, la mise en scène, l'art de l'étalage ", bref, l'amour du bel effet incarné par Wagner. Il voit dans ce « mélange chaotique de tous les styles ${ }^{42}$ " le signe d'une barbarie des " philistins de la culture " qu'il dénonce dans la première Considération Intempestive où il oppose à la pseudoculture de l'imitation de l'art français l'exigence d'une culture allemande originale et féconde dont l'" accumulation grotesque " de tous les styles de l'art de son temps lui semble bien éloignée. L'imitation n'est donc ici que le signe d'une incapacité à créer et d'une médiocrité existentielle du " philistin de la culture " qui réduit l'art à un plaisir raisonnable et sain, mais qui écarte résolument tout excès, toute génialité. Il ne laisse à l'artiste que le choix soit d'une imitation de la réalité " d'une façon aussi simiesque que possible, dans des idylles ou des satires doucement humoristiques, soit de copier librement les œuvres classiques les plus connues et les plus célèbres moyennant quelques concessions pudiques au goût du jour ${ }^{43}$. "Nietzsche dénonce ainsi la fin de l'idéalisme esthétique classique au profit d'un pseudo-romantisme dégradé où le beau, adapté aux goûts de la masse, devient le plaisant, l'agréable et perd toute dimension sublime et transcendante.

39. Yves Michaud note ainsi que "l'art se volatilise en éther esthétique ", l'œuvre disparaissant au profit de dispositifs producteurs d'effets esthétiques, in L'art à l'état gazeux, Paris, Fayard / Pluriel, 1970, p. 8.

40. Gilles Lipovetsky, L'Ėre du vide. Essais sur l'individualisme contemporain, Paris, Gallimard (coll. "Folio "), 1983.

41. Ibid., p. 127.

42. Nietzsche, Considérations intempestives, Paris, Flammarion, 1988, p. 25 / Unzeitgemäße Betrachtungen, Stuttgart, Reclam, p. 24: " ... der Stillosigkeit oder dem chaotischen Durcheinander aller Stile. "

43. Ibid., p. 43 / p. 42 : " Entweder Nachhamung der Wirklichkeit bis zum Aeffischen, in Idyllen oder sanftmütigen humoristischen Satiren, oder freie Kopien der anerkanntesten und berühmtesten Werke der Klassiker. " 


\title{
Le kitsch comme produit d'une culture du loisir et non plus de la contemplation
}

Adorno, dans sa Dialectique de la raison, dénonce en 1944 le mensonge du kitsch organisé par l'" industrie culturelle " qui, en tentant d'annuler l'opposition entre art facile et art sérieux, consacre le mauvais goût en art, notamment transformant en art les objets de consommation ordinaires comme les Soupes Campbell. Broch ${ }^{44}$ analyse quant à lui la faculté d'imitation kitsch comme forme de noyautage; le kitsch, qu'il considère comme le " mal dans le système des valeurs de l'art ", forme son propre système, renfermé sur lui-même, mais n'existe pas sans l'homme kitsch qui, comme producteur, le fabrique et comme consommateur en jouit, préférant un beau plutôt qu'un bon travail et faisant $\mathrm{du}$ bel effet une fin immédiate:

\begin{abstract}
Kitsch könnte weder entstehen noch bestehen, wenn es nicht den Kitschmenschen gäbe, der den Kitsch liebt, ihn als Kunstproduzent erzeugen will und als Kunstkonsument bereit ist, ihn zu kaufen und sogar gut zu bezahlen [...]. Wenn der Kitsch Lüge ist, [...], so fällt der Vorwurf auf den Menschen zurück, der solch Lügen- und Verschönerungsspiegel braucht, um sich daran zu erkennen und mit gewissermaßen ehrlichem Vergnügen sich zu seinen Lügen zu bekennen. ${ }^{45}$
\end{abstract}

Kundera reprend les analyses de Broch ${ }^{46}$ pour voir dans le kitsch une catégorie morale: le kitsch est l'idéal esthétique d'un monde où est niée la " merde ", c'està-dire tout ce qui dans l'Être est contradiction et précarité ; il est un paravent qui dissimule la mort, un miroir embellissant auquel nul n'échappe: " le kitsch exclut de son champ de vision tout ce que l'existence humaine a d'essentiellement inacceptable ${ }^{47}$ ". Mais pour produire l'émotion, il vise à tisser une forme de fraternité en suscitant des sentiments identiques chez le plus grand nombre en recourant à des images clés de la mémoire humaine comme la fille ingrate, la patrie trahie, le souvenir du premier amour ${ }^{48}$.

Le kitsch se rattache donc à l'idylle en tant qu'oubli de l'Être, qu'élimination de ce qui en l'Être est complexe au profit d'un Être simplifié et cohérent, le désir d'embellir le monde se réalisant en rejetant ce qui y résiste. Nourri de la fascination mimétique pour le romantisme et pour l'esthétisme fin de siècle, le kitsch peut

44. Hermann Broch, "Einige Bemerkungen zum Problem des Kitsches », 1950, in Dichten und Erkennen 1, Zürich, Rhein Verlag, 1955 / Quelques remarques à propos du kitsch, Paris, Allia, 2001.

45. Ibid., p. 295.

46. Musil et Broch opposent à la notion de kitsch (fondé sur un manque d'imagination esthétique et tourné vers l'esthétisme et l'industrie du divertissement) celle d'héroïsme. Catherine Coquio souligne quant à elle la puissance mortifère du kitsch: " en empruntant, le kitsch simplifie, pétrifie et dévitalise " (p. 321) ; "l'art pour l'art honore un Beau qui se fige dans le kitsch à chaque chute de tension. Le kitsch est le spleen de l'art moderne " ( Kitsch et critiques du kitsch ", p. 321).

47. Milan Kundera, L'insoutenable légèreté de l'être, Paris, Gallimard (coll. "Folio "), 1989, p. 356-357.

48. Ibid., p. 362-363. 
ainsi créer une forme de totalitarisme de l'idylle en instrumentalisant le bel effet pour maintenir l'ordre existant. Il reste pour cette raison, note Kundera, le meilleur soutien des hommes politiques, notamment des régimes totalitaires ${ }^{49}$. Rejetant toute discordance, toute distance ironique, toute manifestation d'individualisme, il vise à figer une image harmonieuse: ainsi les scènes où Hitler embrasse un enfant, les hagiographies des dictateurs, ou les photographies d'une Leni Riefensthal présentant des corps glabres et athlétiques comme autant de miroirs de la force de l'Allemagne hitlérienne. Car le kitsch exclut tout questionnement critique en présentant l'image d'un microcosme fermé sur ses convictions. " $\mathrm{Au}$ royaume du kitsch totalitaire, les réponses sont données d'avance et excluent toute question nouvelle. Il en découle que le véritable adversaire $d u$ kitsch totalitaire, c'est l'homme qui interroge ${ }^{50}$ ", affirme Kundera. Dans son roman, il évoque ainsi la révolte du personnage de Sabina, artiste peintre dont le régime communiste vante les mérites et le " combat pour la liberté» pour mieux désamorcer par ce kitsch de propagande sa révolte potentielle ; elle s'écrie avec rage: " mon ennemi, ce n'est pas le communisme, c'est le kitsch ! ", le kitsch né de ce mensonge politique par lequel les gens veulent "fabriquer leur (?) vie ${ }^{51}$." Qu'il dénote une soif de sécurité, un instinct de possession et de jouissance immédiate, un refuge dans l'idylle ou dans la consolation de la belle apparence, le kitsch est donc une forme d'illusion entretenue; mais nul ne peut y échapper car " le kitsch fait partie de la condition humaine ${ }^{52}$ ".

Quoi qu'il en soit, le kitsch est donc associé à la décadence. Jankélévitch, en $1950^{53}$, en fait le symptôme de la " conscience décadente ", y voyant une maladie infantile de l'art; Adorno y voit quant à lui un signe de dérive de l'esthétisme et un art de l'inauthentique car privé de son autonomie en se bornant à garantir une " jouissance culinaire " individuelle :

Sinnliche Wohlgefälligkeit [...] ist geschichtlich zum unmittelbar kunstfeindlichen geworden, Wohllaut des Klangs, Harmonie der Farben, Suavität zum Kitsch und zur Kennmarke der Kulturindustrie. ${ }^{54}$

Mais, niant sa propre dimension sociocritique, le kitsch ne peut prétendre au statut d'art sérieux et autonome:

Eines der Momente von Kitsch.... wäre die Vortäuschung nicht vorhandener Gefühle und damit deren Neutralisierung... Kitsch wäre die Kunst, die nicht ernst genommen werden will und die doch durch ihr Erscheinen ästhetischen Ernst postuliert [...]. Kitsch ist sowohl

49. Dans Masse et puissance, Canetti décrit ainsi un cortège civil à Nuremberg, où des sections se succèdent au pas cadencé, drapeaux portés à l'épaule, surchargées de figures héraldiques, leurs costumes évoquant un retour romantique au Moyen Âge et niant toute forme de temporalité. On retrouve une même dénonciation de ce gigantisme et de ce culte du bel effet kitsch dans Le Tambour de Grass.

50. Kundera, L'insoutenable légèreté de l'être, p. 368.

51. Ibid., p. 368-369.

52. Ibid., p. 372.

53. Vladimir Jankélévitch, "La décadence ", Revue de métaphysique et de morale 4, 1950, p 337369.

54. Ibid., p. 411. 
von Kunst qualitativ verschieden wie deren Wucherung, präformiert in dem Widerspruch, dass autonome Kunst über die mimetischen Impulse verfügen muss, die solcher Verfügung entgegen sind. ${ }^{55}$

L'art kitsch s'oppose de fait à la conception adornienne d'un art critique dénonçant les méfaits de l'» industrie de la culture " et du capitalisme:

Nur Kunstwerke, die als Verhaltensweise zu spüren sind, haben ihre raison d'être. Kunst ist nicht nur der Statthalter einer besseren Praxis als der bis heute herrschenden, sondern ebenso Kritik von Praxis als der Herrschaft brutaler Selbsterhaltung inmitten des Bestehenden ${ }^{56}$.

Si le kitsch est conçu comme négation du réel et de l'histoire, il court enfin le risque de la sclérose et de la vacuité:

Plus l'œuvre s'interdit la conscience de la mort, plus vite elle court à la sienne... Produit d'une glissade maladroite du principe de plaisir sur la réalité, le kitsch condamne le sublime à la vulgarité, la fresque au graffiti, le refoulé à son retour. Administrée comme en antidote à une réalité décrétée laide, l'idée du Beau se livre au canon et à l'ornement, et se rend ainsi vulnérable à cette réalité même ${ }^{57}$.

\section{Le kitsch comme jeu et comme arme}

Dans la seconde moitié du XXe siècle, notamment entre 1950-1960, paraissent aux Etats-Unis et en Italie nombre d'ouvrages critiques sur l'art de masse et l'évolution du goût. C'est alors que s'opère un tournant, le kitsch, jadis défini comme enfer de l'art, devenant un principe de jeu, de déconstruction des normes du bon goût, de développement d'une culture populaire; on valorise également le kitsch au second degré, le kitsch citationnel, comme caractéristique de la culture postmoderne.

Il y a de fait un plaisir indéniable du kitsch, plaisir régressif, image du temps perdu, " mémoire suspendue " que Céleste Olalquiaga définit comme " rebut témoignant de ce qui n'est plus, une bulle de temps, billet aller-retour pour le pays du mythe des rêves collectifs et individuels ${ }^{58}$ ". Nul hasard non plus si un des cadres privilégiés du kitsch sont les passages parisiens construits entre 1780 et 1860 qui accompagnent le déclin de l'aura en inventant la flânerie et le lèche-vitrines, en offrant avec un luxe de miroirs, de verre et de métal un refuge loin du chaos de la rue et une image de rêve préservée des atteintes du temps, que Benjamin, après l'Aragon du Paysan de Paris, définit comme un " aquarium de vie primitive ", une fantasmagorie régressive offrant à l'homme des villes un paysage intérieur onirique. Le kitsch cristalliserait en ce sens le goût du merveilleux, la quête d'un cocon doux et protecteur, le retour au monde englouti de l'enfance; c'est aussi ce qui explique la vogue kitsch du Crystal Palace ou

55. Theodor W. Adorno, Ästhetische Theorie, Frankfurt a. M., Suhrkamp, 2000, p. 466-467.

56. Ibid., p. 26.

57. Coquio, "Kitsch et critiques du kitsch ", p. 324.

58. Olalquiaga, Royaume de l'artifice, p. 26. 
des grottes artificielles dont la plus célèbre est évidemment la grotte de Vénus à Linderhof.

C'est aussi cet amour enfantin du clinquant que confesse Rimbaud lorsqu'il évoque dans Une saison en enfer son goût passé pour les " refrains niais " les " peintures idiotes ", les " toiles de saltimbanques ou les " enluminures populaires $^{59}$ ", ou lorsque Baudelaire mentionne le magasin de madame Panckouke, sa " fée du joujou ", et sa chambre aux trésors de jouets de pacotille, " inextricable fouillis de formes bizarres et de couleurs disparates ${ }^{60}$ ".

Il peut enfin exister un élitisme du kitsch lorsqu'il désigne une pratique artistique comme celle d'Andy Warhol ou de Jeff Koons. C'est au nom du plaisir fétichiste que Jeff Koons, ancien trader considérant l'art comme marchandise, comprend que la première des motivations d'achat est la fétichisation de l'objet d'art; érigeant, dans la continuité des ready-made de Duchamp, la banalité en œuvre d'art, réintroduisant le quotidien dans l'art, dans Michael Jackson and bubbles, sa guenon, il réhabilite l'ornement, le caprice, la légèreté d'un quotidien réenchanté par son imitation et son grossissement ludique. Tout en reliant " l'étude de l'objet d'art à celle de ses conditions de production et de consommation $^{61}$ ", sans inventer de nouvelles formes, Koons, en représentant des idoles livrées à l'adoration des spectateurs, dénonce en même temps la " cour du simulacre ", consacrant certes le fétiche, mais faisant de l'objet " le lieu d'inscription, de réification, de sédimentation du sens, et donc également le lieu de sa critique ${ }^{62} "$.

Il importe donc de distinguer le kitsch involontaire du kitsch volontaire, produit d'une intention artistique. Si le kitsch involontaire " produit du laid à force de vouloir le beau ${ }^{63}$ ", le kitsch volontaire invente un beau inconnu, " bizarre ", moderne au sens baudelairien du terme, en mimant le contretemps kitsch pour faire signifier une laideur assumée, la laideur industrielle, reproduite sur un mode réflexif et ironique, dans une volonté de transgression par l'art de la transgression du beau. L'art moderne peut ainsi jouer de la perte de l'aura de l'œuvre d'art en " intégrant sa dissonance dans une esthétique du Beau relatif, passager, historique en fait des détritus de la laideur bourgeoise ${ }^{64}$ ". Le kitsch incarnerait en ce sens un art de l'anti-renoncement ${ }^{65}$ à l'idéal d'un Beau que l'on sait perdu, mais dont on maintient le regret.

59. Arthur Rimbaud, Alchimie du verbe, in Une Saison en enfer, Paris, Gallimard (coll " Poésies "), 1965, p. 139.

60. Baudelaire, Morale du joujou, in Écrits sur l'art, Paris, Le Livre de Poche, 1992 et 1999, p. 245.

61. Coquio, "Kitsch et critiques du kitsch ", 314.

62. Isabelle Alfandary, "L'insoutenable légèreté des objets dans l'œuvre de Jeff Koons », in MarieChristine Lemardeley, Didier Topia (dir.), L'Empreinte des choses, Paris, Presses Sorbonne Nouvelle, 2007, p. 172.

63. Coquio, "Kitsch et critiques du kitsch ", p. 315.

64. Ibid., p. 315 sq.

65. Ibid., p. 315: "Le kitsch est ce qui reste de l'absolu lorsque la fonction sociale de l'art semble faire défaut. Et ce qui reste, c'(est) la foi dans le Beau... " 
Enfin, n'oublions pas le jeu postmoderne avec le kitsch lorsqu'il se cite et s'amuse de lui-même au sein de l'œuvre d'art, dans l'ironie et la citation kitsch par exemple. Ainsi Kundera fait-il dire au personnage Sabina dans L'Insoutenable légèreté de l'Être:

Toute sa vie, elle a affirmé que son ennemi c'est le kitsch. Mais est-ce qu'elle ne le porte pas elle-même au fond de son être? Son kitsch, c'est la vision d'un foyer paisible, doux, harmonieux, où règnent une mère aimante et un père plein de sagesse [...]. Elle a senti plus d'une fois ses yeux s'humecter en voyant à la télévision, dans un film sentimental, une fille ingrate serrant dans ses bras un père abandonné.

Si le narrateur reprend ici des images kitsch, c'est pour les déjouer par l'ironie, démontant ainsi le mythe de la famille idéale. Chez Jelinek aussi, le recours au kitsch au second degré a pour vocation de démythifier l'amour et d'en dénoncer les clichés aliénants. Dans Les Amantes, le kitsch est fondé sur la répétition du même dont la monotonie devient mortifère et témoigne de la perte de langage de la femme tout en dénonçant la belle apparence trompeuse de l'idylle autrichienne. Le roman s'ouvre en effet sur une évocation trop idyllique pour être vraie :

Kennen Sie dieses SCHÖNE land mit seinen tälern und hügeln? (...) Kennen Sie seine friedlichen häuser und die friedlichen menschen darinnen ${ }^{66}$ ?

L'incipit du roman le présente donc d'emblée comme un roman kitsch au sens où l'entend Hermann Broch, " Der Kitschroman (schildert) die Welt nicht, „wie sie wirklich ist“ sondern, „wie sie gewünscht oder gefürchtet wird ${ }^{670 . " ~ " ~}$ Les protagonistes du roman, Paula et Brigitte, se repaissent elles-mêmes de romans kitsch, romans de terroir ou romans feuilletons, qui nourrissent leurs fantasmes stéréotypés du prince charmant et du bonheur conjugal petit-bourgeois :

Dann will ich mir einen braven mann suchen [...] und da will ich heiraten und kinder bekommen. Une alle miteinander und auch noch zugleich lieben, ja, lieben! und es sollen zwei sein, ein bub und ein mädel [...] und alles immer sauber und rein ${ }^{68}$.

Mais c'est dans l'évocation du mariage des deux femmes que la parodie du style kitsch des romans d'amour nourri de l'imitation servile de clichés et du culte du bel effet trompeur atteint son point culminant: "Heute ist endlich der ersehnte tag gekommen. Strahlendblaues wetter begrüßt den langersehnten tag ${ }^{69}$ " Si c'est ici la " perspective avec " qui est adoptée, c'est pour mieux ironiser sur le beau mensonge dont s'abreuvent ces femmes qui finiront par être victimes du pouvoir patriarcal et d'un mythe amoureux glorifiant selon Barthes " la clôture du foyer, son introversion pantouflarde ${ }^{70}$ ", infantilisant et coupant la femme de toute responsabilité sociale: le recours au style kitsch est donc ici ironique et vise à démystifier le mythe de l'amour. Dans le cas de Jelinek comme des auteurs

66. Elfriede Jelinek, Die Liebhaberinnen, Roman, Reinbek bei Hamburg, rororo, 1975, p. 5.

67. Hermann Broch, " Einige Bemerkungen zum Problem des Kitsches, Ein Vortrag ", in Dichten und Erkennen. Essays, vol. I, Zürich, Rhein Verlag, 1955, p. 344.

68. Jelinek, Die Liebhaberinnen, p. 19.

69. Ibid., p. 136.

70. Roland Barthes, Mythologies, Paris, Seuil, 1957, p. 46. 
qui recourent au kitsch comme symptôme, le kitsch n'est pas une " perte de sens, mais un choc de significations.... Une nappe de sens venant surgir dans les sillons d'un autre pour dire autre chose autrement ${ }^{71}$ ". Lorsqu'il dénonce la laideur sociale, il peut ainsi devenir ainsi l'instrument d'une " discrète et radicale critique politique ${ }^{72}$ ".

Pour conclure, soulignons avec Christophe Genin que, "si le kitsch fut initialement l'objet d'un jugement péjoratif, il est peu à peu devenu un style artistique apprécié, assumé $^{73}$ " : il y a donc une réversibilité du kitsch qui, d'art de la médiocrité et de symptôme de décadence, est devenu peu à peu " tendance ", qu'il soit promu comme principe de plaisir, de démocratisation du beau, comme jeu esthétique ou comme vecteur de critique sociale. Reste que le kitsch est bien une figure de l'ambiguïté qui se rit de la gravité de l'art ancien; il peut être euphorisant en présentant un monde enchanté, apportant le soulagement et le confort après la terreur, même si cette forme d'idylle peut résulter d'un effacement de la mémoire ou du refoulement d'une réalité niée. Certes, le kitsch associe le beau non au vrai ou au bien, mais à l'effet; certes, son refuge dans l'idylle peut viser à exclure tout ce que l'existence humaine a d'essentiellement inacceptable (Kundera), ce masque de la belle apparence occultant la dimension tragique de l'existence ${ }^{74}$. Mais ne peut-on pas voir aussi dans ce processus de refoulement de la souffrance et dans cette fascination pour le beau multiple, pour la copie rassurante, un " truc " " humain trop humain " un " miroir destructible certainement, fallacieux probablement, mais humain pleinement ${ }^{75}$ " témoignant notre attachement à l'» insoutenable légèreté de l'être »?

71. Genin, Kitsch dans l'âme, p. 78.

72. Coquio, "Kitsch et critiques du kitsch ", p. 316.

73. Genin, Kitsch dans l'âme, p. 7.

74. Dans Weiter leben (1992) / Refus de témoigner (1997), et dans des conférences tenues à l'université de Bonn en 1994-1995 ("Missbrauch der Erinnerung: KZ-Kitsch ", Von hoher und niedriger Literatur, Göttingen, Wallstein, 1996), Ruth Klüger récuse ainsi le " kitsch concentrationnaire " et le "Kitsch muséal " dans les stéréotypes engendrés par l'" abus de mémoire " de la Shoah et qui visent à travestir la réalité vécue, laquelle reste de l'ordre de l'indicible. Voir article de C. Coquio, "Envoyer les fantômes au musée? " Critique du "kitsch concentrationnaire " par deux rescapés: Ruth Klüger, Imre Kertés, Gradhiva 2007/1 (n5), en ligne: [http ://www.cairn.info/revue-gradhiva-2007-1-page-4.htm].

75. Genin, Kitsch dans l'âme, p. 124. 
